\title{
Espironolactona aumenta los niveles circulantes de angiotensina-(1-9) y revierte el remodelado cardíaco en la hipertensión arterial experimental
}

María Paz Ocaranza, Jackeline Moya, Patricio Araos, David Mondaca, Jorge E Jalil

División de Enfermedades Cardiovasculares, Pontificia Universidad Católica de Chile.

Financiamiento: Fondef D1111122 (MPO, JEJ), FONDECYT 1085208 y 1121060 (JEJ, MPO) y FONDAP 15130011 (MPO)

Resumen: Previamente hemos demostrado que la eficacia de enalapril, candesartán y de fasudil -inhibidor de la vía RhoA/ROCK,- en el tratamiento del remodelado cardíaco en la hipertensión (HTA) e infarto al miocardio está mediada por aumento en los niveles circulantes del péptido vasoactivo angiotensina [Ang](1-9). Sin embargo, no hay información disponible si el antagonista del receptor de mineralocorticoide, espironolactona (espiro) disminuye el remodelado cardíaco aumentando los niveles circulantes de Ang-(1-9). El objetivo de este trabajo fue determinar si espironolactona disminuye el remodelado cardíaco aumentando los niveles circulantes de Ang-(1-9) en la hipertensión arterial experimental.

Métodos. Estudio comparativo de 3 grupos experimentales. Se utilizaron ratas Sprague Dawley macho (150 \pm 10 grs) unifrectomizadas tratadas con desoxicorticosterona (DOCA, $60 \mathrm{mg} / \mathrm{Kg} 2$ veces sem, im) por 6 semanas. Como controles (Sham) se usaron ratas unifrectomizadas. A partir de la $3^{\circ}$ semana las ratas DOCA con HTA $>140 \mathrm{mmHg}$ fueron randomizadas a recibir vehículo o espiro (100 mg/kg día, gavage) por $3 \mathrm{sem}$. Al finalizar el tratamiento se determinó la presión arterial sistólica (PAS), masa corporal, peso del corazón (PC) y masa cardíaca relativa al largo de la tibia (MCR, mg ventrículos/LT*100). El grado de hipertrofia cardiomiocitaria se determinó midiendo el área y perímetro de los cardiomiocitos y la fibrosis por el contenido de colágeno en cortes teñidos con rojo picrosirio. Re- sultados (promedio $\pm \mathrm{ES}$ ):

\begin{tabular}{|l|c|c|c|}
\hline N & Sham & DOCA & DOCA+Espiro \\
\hline MC $(g)$ & 8 & 8 & 8 \\
\hline PAS $(\mathrm{mmHg})$ & $414(29)^{*}$ & $351(17)$ & $322(11)$ \\
\hline MCR (mg/cm) & $119(7)$ & $198(11)^{* *}$ & $126(1) \#$ \\
\hline Area cardiomiocitos (um2) & $231(1)$ & $537(4)^{* *}$ & $312(1) \#$ \\
\hline Colágeno cardíaco (\%) & $2.9(0.1)$ & $16.5(1.1)^{* *}$ & $4.7(0.4) \#$ \\
\hline Ang-(1-9) pg/mL & $46.4(1.4)$ & $41.7(7.1)$ & $221.1(51.8) \#$ \\
\hline * $p<0.05$ vs DOCA y DOCA+Espiro,${ }^{* *} p<0.05$ vs Sham, \#p<0.05 vs DOCA & $1.80(0.80)^{* *}$ & $0.36(0.01) \#$ \\
\hline \begin{tabular}{l} 
y Sham (post-ANOVA). \\
\hline
\end{tabular}
\end{tabular}

Conclusión: Espironolactona disminuye la PAS y aumenta los niveles circulantes de Ang-(1-9). Este aumento en los niveles circulantes de Ang-(1-9) se asocia con una disminución significativa de la hipertrofia y la fibrosis cardiaca hipertensiva. Este nuevo efecto de espironolactona en los niveles circulantes de Ang-(19), - péptido vasoactivo de la vía paralela del sistema renina-angiotensina-aldosterona,- podría contribuir al efecto antihipertensivo y disminución del daño cardiaco en la hipertensión y remodelamiento cardiovascular y renal patológico. Estos hallazgos pueden tener relevancia terapéutica en términos que Ang-(1-9) podría disminuir el daño cardiovascular patológico.

\section{Correspondencia:}

Dra. María Paz Ocaranza,

Departamento de Enfermedades Cardiovasculares.

Facultad de Medicina. P. Universidad Católica de Chile.

Tel: +562-23543407, Fax: +562-26321924.

mocaran@med.puc.cl 


\section{Spironolactone increases circulating levels of Angiotensin-(1-9) and reverts cardiac remodeling in experimental hypertension}

We have previously demonstrated that the efficacy of enalapril, candesartan and fasudil,- RhoA / ROCK inhibitor-, in the treatment of cardiac remodeling in hypertension (HT) and myocardial infarction is mediated by an increase in circulating levels of the vasoactive peptide angiotensin (Ang) -(1-9). However, it is not known whether the mineralocorticoid receptor antagonist, spironolactone (spiro) decreases cardiac remodeling by increasing the circulating levels of Ang- (1-9). The aim of this study was to determine whether spironolactone decreases cardiac remodeling by increasing circulating levels of Ang(1-9) in experimental hypertension.

Methods. Comparative study of 3 experimental groups. Unifirectomized male Sprague Dawley rats $(150 \pm 10$ grams) were treated with deoxycorticosterone (DOCA, $60 \mathrm{mg} / \mathrm{kg} 2$ times a week, im) for 6 weeks. Unifirectomized rats were used as controls (Sham). At 3rd week after surgery, DOCA rats with HTA $>140 \mathrm{mmHg}$ were randomized to receive vehicle or spironolactone (Spiro, $100 \mathrm{mg} / \mathrm{kg}$ day, gavage) for 3 weeks. At the end of treatment, systolic blood pressure (SBP), body mass (BM), heart weight $(\mathrm{HW})$ and relative cardiac mass to the tibia length (MCR, mg ventricles / LT * 100) were determined. The degree of cardiomyocyte hypertrophy was determined by measuring the area and perimeter of cardiomyocytes and fibrosis by collagen content in sections stained with picrosirius red.

Results (mean $\pm \mathrm{ES}$ ):

\begin{tabular}{|l|c|c|c|}
\hline N & Sham & DOCA & DOCA+ Spiro \\
\hline BM $(g)$ & 8 & 8 & 8 \\
\hline SBP $(\mathrm{mmHg})$ & $414(29)^{*}$ & $351(17)$ & $322(11)$ \\
\hline RCM (mg/cm) & $119(7)$ & $198(11)^{* \star}$ & $126(1) \#$ \\
\hline Cardiomyocytes' (um2) & $0.29(0.01)$ & $1.80(0.80)^{* *}$ & $0.36(0.01) \#$ \\
\hline Cardiac Collagen (\%) & $231(1)$ & $537(4)^{* *}$ & $312(1) \#$ \\
\hline Ang-(1-9) pg/mL & $46.4(1.4)$ & $16.5(1.1)^{* *}$ & $4.7(0.4) \#$ \\
\hline *p< 0.05 vs DOCA y DOCA+Spiro, ${ }^{* *}$ p $<0.05$ vs Sam, \#p<0.05 vs \\
\hline DOCA y Sham (post-ANOVA) & & \\
\hline
\end{tabular}

Conclusion: Spironolactone decreases systolic blood pressure and increases circulating levels of Ang(1-9). This increase in circulating levels of Ang- (1-9) was associated with a significant decrease in hypertrophy and hypertensive cardiac fibrosis. This new effect of spironolactone on the circulating levels of Ang- (1-9) - vasoactive peptide of the parallel pathway of the renin-angiotensin-aldosterone system - could contribute to the antihypertensive effect and decrease of cardiac damage in HT and cardiovascular remodeling and renal disease. These findings may have therapeutic relevance supporting that Ang-(1-9) may decreases pathologic cardiovascular damage.

Keywords: spironolactone; aldosterone; renin angiotensin aldosterone system; hypertension; angiotensin-(1-9); cardiac remodeling. 


\section{Introducción:}

El tratamiento antihipertensivo actual, enfocado sobre diversas vías y mecanismos fisiopatológicos y celulares que regulan y normalizan la presión arterial elevada, disminuye la progresión del daño de órganos blanco y reduce en forma muy importante la incidencia de accidentes cerebrovasculares, de insuficiencia cardíaca y de insuficiencia renal. Sin embargo, con los mejores tratamientos antihipertensivos disponibles, en los pacientes hipertensos con mayor riesgo y remodelado cardiovascular (CV), este riesgo no se modifica sustancialmente a pesar de reducir las cifras de presión arterial y se le ha denominado también riesgo residual en la hipertensión (HTA). ${ }^{1}$

Por esta razón, en la actualidad uno de los desafíos terapéuticos importantes en la HTA está en identificar mecanismos que además de normalizar las cifras tensionales produzcan remodelado reverso con mayor eficacia respecto del tratamiento actual. En este sentido, el recientemente descubierto eje fisiopatológico constituido por la enzima convertidora de angiotensina I homóloga (ECA2), angiotensina [Ang]-(1-7), Ang-(1-9) y alamandina, contrarresta los efectos vasoconstrictores, retenedores de sodio y pro remodelado del eje clásico renina-Ang II-aldosterona (SRAA) y podría constituir una alternativa más eficaz en el tratamiento de la HTA. ${ }^{2}$

El nonapéptido endógeno Ang-(1-9) se produce a través de la hidrólisis de Ang I por acción de la enzima ECA2 y puede hidrolizarse a Ang-(1-7) por acción de la ECA. ${ }^{3}$ En comparación con Ang-(1-7) - que posee un receptor específico, el receptor Mas -, Ang-(1-9) tiene una vida media más prolongada. ${ }^{4,5}$

Previamente, observamos que la administración de Ang(1-9) redujo la presión arterial (PA) en ratas hipertensas por infusión de Ang II y también en el modelo de Goldblatt. ${ }^{6}$ Ang-(19) además mejoró significativamente la función diastólica del ventrículo izquierdo (VI, evaluada con ecocardiografía) y la función endotelial en arterias mesentéricas de pequeño diámetro. Además, disminuyó la hipertrofia cardíaca y de la pared y aórtica, la fibrosis, el estrés oxidativo, el colágeno y los niveles del factor de crecimiento transformante $\beta$ - 1 en aorta y en la pared del VI. ${ }^{6}$ En los experimentos mencionados, los efectos antihipertensivo y anti remodelado de Ang-(1-9) fueron mitigados por la administración conjunta del bloqueador del receptor de angiotensina I tipo 2 PD123319, pero no por la coadministración del antagonista del receptor Mas A779 (el receptor de angiotensina 1-7), ${ }^{4}$ lo que señala que Ang (1-9) no actúa a través del receptor Mas. La administración de Ang-(1-9) también disminuyó los niveles de Ang II, la actividad de la ECA y el estrés oxidativo en la pared aórtica y el ventrículo izquierdo y aumentó los niveles mRNA de la óxido nítrico sintasa endotelial en la aorta, así como los niveles de nitrato en plasma. ${ }^{4}$

Por otro lado, desde el punto de vista clínico, además del sistema simpático y del SRAA, el rol de aldosterona y del bloqueo del receptor de mineralocorticoide en el tratamiento de la HTA es cada vez más relevante. En el reciente estudio randomizado a doble ciego Pathway-2 (ensayo cruzado, controlado con placebo) en pacientes hipertensos resistentes a 3 fármacos (IECA/ARAII, calcio antagonista y una tiazida), todos los pacientes recibieron espironolactona (Espiro, 25-50 mg/d), doxazosina de liberación prolongada (4-8 mg/d), y bisoprolol (5-10 $\mathrm{mg} / \mathrm{d}$ ), en forma secuencial durante 12 semanas cada uno. ${ }^{7}$ Los resultados mostraron que espironolactona fue mucho más efectiva que los otros 2 fármacos en reducir la presión arterial en pacientes hipertensos resistentes con el esquema $\mathrm{A}+\mathrm{C}+\mathrm{D}$ de base $^{7}$, actuando principalmente a través de la retención de sodio en estos pacientes.

Al momento no existe información respecto del efecto de espironolactona sobre los niveles de Ang (1-9) en la HTA. En el presente trabajo hemos planteado la hipótesis de que los niveles de Ang (1-9) aumentan con espironolactona lo que podría constituir un mecanismo antihipertensivo y de anti remodelado cardiovascular adicional de este grupo de fármacos. De acuerdo con lo anterior el objetivo de este estudio fue determinar si espironolactona aumenta los niveles circulantes de Ang-(1-9) a la par que disminuye el remodelado cardiaco en la hipertensión arterial experimental.

\section{Métodos:}

La presente investigación corresponde a un subestudio dentro de un trabajo orientado a evaluar el efecto de los diuréticos sobre la activación de Rho kinasa y la expresión de genes que promueven el remodelado y estrés oxidativo a nivel tisular en la pared aórtica de ratas hiperten$\operatorname{sas}^{8}$ y del que se ha conservado plasma, suero y tejidos para análisis posteriores.

Este estudio en animales, de realizó de acuerdo con la "Guía para el cuidado y uso de animales de laboratorio" publicada por el "National Hatch Instituye" (NIH N ${ }^{\circ} 85-$ $23,1985)$ y fue aprobada por la Comisión de Investigación de la Facultad de Medicina de la Pontificia Universidad Católica de Chile.

1.- Modelo experimental. Se utilizaron ratas Sprague Dawley macho $(150 \pm 10$ grs $)$ unifrectomizadas tratadas 
con desoxicorticosterona (DOCA, 60mg/Kg 2 veces por semana, im) por 6 semanas. Como controles (Sham) se usaron ratas unifrectomizadas. A partir de la $3^{\circ}$ semana las ratas DOCA con HTA> $140 \mathrm{mmHg}$ fueron randomizadas a recibir vehículo o espiro (100 mg/kg día, gavage) por 3 semanas. Al finalizar el tratamiento se determinó la presión arterial sistólica (PAS) y masa corporal (MC). Posteriormente los animales fueron sacrificados. ${ }^{8}$

2.- Presión arterial sistólica (PAS). Se utilizó el método pletismográfico en la cola de los animales una vez por semana por investigadores ciegos al tratamiento. ${ }^{9}$

3.- Obtención del tejido ventricular: Los animales fueron anestesiados con $\mathrm{HCl} /$ xilazina [35 y $7 \mathrm{mg} / \mathrm{kg}$ i.p., respectivamente]. El corazón fue extraído y lavado en suero fisiológico, donde una parte del ventrículo fue fijado en Bouin por $12 \mathrm{~h}$ y posteriormente incluido en parafina. $\mathrm{El}$ resto del tejido fue conservado a $-80^{\circ} \mathrm{C} .{ }^{10}$

4.- Evaluación de la hipertrofia cardíaca. Se evaluó a). Masa cardíaca relativa $(\mathrm{MCR}, \mathrm{g} / \mathrm{cm})$, mediante la relación entre la masa del corazón ( $\mathrm{g}$ ) excluidas ambas aurículas) y el largo de la tibia $(\mathrm{LT}, \mathrm{cm}) .{ }^{8} \mathrm{~b}$ ). Morfometría de cardiomiocitos. Cortes transversales de la porción media del corazón (ambos ventrículos) de $5 \mu \mathrm{m}$ grosor fueron teñidos con tinción de hematoxilina-eosina, para ser analizados mediante microscopía de luz (Nikon Eclipse E400). Las imágenes fueron obtenidas con cámara Nikon DS Fi1 y proyectadas a un monitor para su posterior análisis mediante el software NIS-Element BR en el cual se midió el área $(\mu \mathrm{m} 2)$ y perímetro $(\mu \mathrm{m})$ de los cardiomiocitos. Se analizaron al menos 100 células por animal de estudio, las cuales se ingresaron como células individuales al análisis estadístico. ${ }^{10}$

\section{5.- Evaluación de la Fibrosis del ventrículo izquierdo}

(VI). Para evaluar la fibrosis intersticial, cortes transversales de la porción media del corazón de $5 \mu \mathrm{m}$ grosor fueron teñidos con rojo picrosirio (Sirius Red $0.1 \%$ en ácido pícrico saturado), para ser analizados con microscopía de luz (Nikon Eclipse E400). Las imágenes fueron captadas con cámara Nikon DS Fi1 y proyectadas a un monitor para la obtención de las imágenes mediante el software NIS-Element BR. Para la evaluación de la fibrosis, el VI se dividió en 2 zonas para el análisis: miocardio y subendocardio, en los cuales se cuantificó el porcentaje de colágeno presente en estas zonas con un software computacional en plataforma Matlab con una aplicación diseñada exclusivamente para este análisis. Para el porcentaje de colágeno miocárdico se analizaron al menos 32 imágenes de la zona por animal de estudio. De igual manera, para determinar el porcentaje de colágeno subendocárdico se analizaron al menos 20 imágenes de la zona por cada animal de estudio. ${ }^{11}$ El porcentaje de colágeno total se determinó mediante la suma del \% de colágeno miocárdico $+\%$ de colágeno subendocárdico. ${ }^{11}$

6.- Niveles circulantes de Ang II y Ang-(1-9): Ambos péptidos se midieron por medio de un kit de ELISA comercial.

7. Análisis estadístico: Los datos obtenidos se expresaron como promedio $\pm \mathrm{DE}$. Los grupos experimentales fueron constituidos por 8 animales por grupo experimental. Para las comparaciones se usó análisis de varianza de una vía (ANOVA) seguido de prueba Newman-Keuls. Análisis de correlaciones se realizaron por el método de Pearson. Se utilizó el programa estadístico SPSS 20.0. Un valor de $\mathrm{p}<0,05$ fue considerado como estadísticamente significativo.

\section{Resultados:}

1.- PAS y masa corporal (MC). En las ratas del grupo control unifrectomizado se obtuvo un promedio de PAS de $119 \pm 7 \mathrm{mmHg}$ (Tabla 1). Las ratas DOCA mostraron un aumento significativo de la PAS (198 \pm 11$)$, respecto al grupo control sham (119 \pm 7 , Tabla 1). Las ratas hipertensas DOCA que recibieron espiro mostraron una disminución significativa de la PAS vs las ratas DOCA+vehículo (126 \pm 1 vs. $198 \pm 11$, respectivamente). La espiro administrada a partir de la tercera semana post cirugía disminuyó significativamente la PAS. Sin embargo, espiro no logró disminuir la PAS de las ratas DOCA al nivel de la PAS de las ratas sham (Tabla 1). La MC mostró diferencias significativas entre los grupos experimentales DOCA+

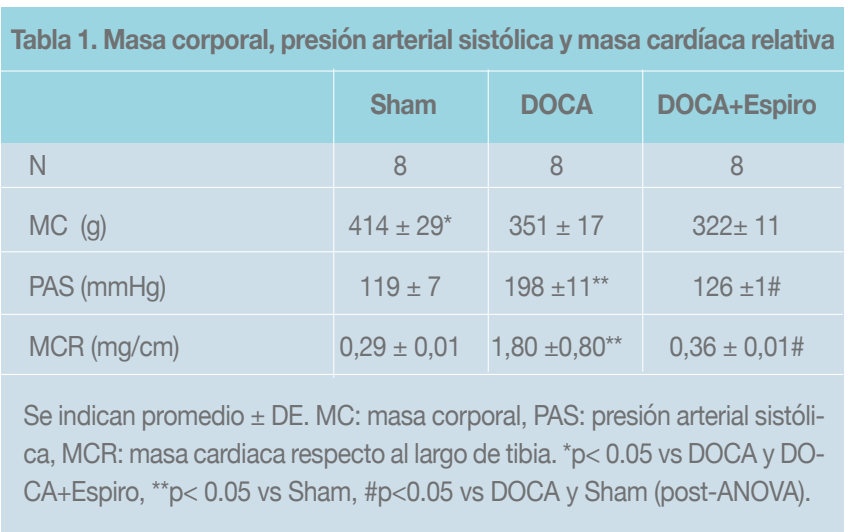


vehículo y DOCA + Espiro versus Sham (Tabla I). El tratamiento con espiro no mejoró la caída de la MC de las ratas DOCA (Tabla I).

2.- Hipertrofia cardíaca. A). La MCR al largo de la tibia fue significativamente superior en el grupo DOCA respecto al grupo Sham $(1,80 \pm 0,80$ vs $0,29 \pm 0,01 \mathrm{gr}$, $\mathrm{p}<0,05)$. El tratamiento con espiro logró regresar significativamente la hipertrofia cardíaca de las ratas DOCA $(0,36 \pm 0,01$ vs $1,80 \pm 0.8, \mathrm{p}<0.05$, respectivamente) Espiro no logró disminuir la MCR al nivel de las ratas controles (Tabla I). b) Hipertrofia cardiomiocitaria. En las ratas hipertensas DOCA se observó un aumento significativo en el área y perímetro de los cardiomiocitos respecto a las ratas controles sham (Figura 1). El tratamiento con espiro logró disminuir la hipertrofia del cardiomiocito, sin embargo, esta disminución no logró alcanzar el área y perímetro de las ratas sham. (Figura 1).

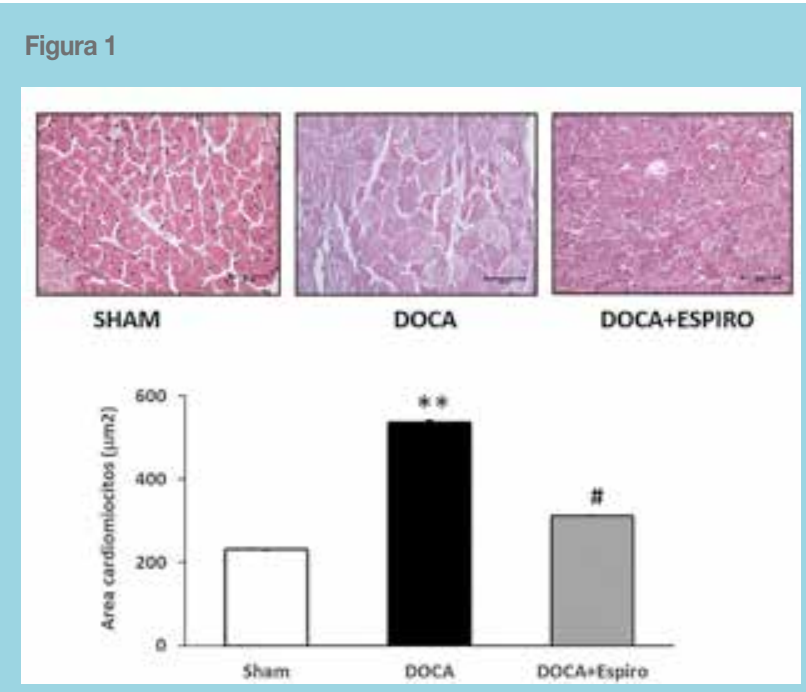

Espironolactona disminuye la hipertrofia cardiomiocitaria en la hipertensión arterial experimental. Ratas hipertensas renina independiente recibieron vehículo o espironolactona por 3 semanas tal como se describe en Materiales y Métodos. Como controles se usaron ratas sham (operación ficticia) (a). Cortes transversales representativos de corazón teñidos con hematoxilina- eosina $(400 \mathrm{X}$, escala barra $50 \mathrm{~mm}$ ) de ratas hipertensa+ vehículo, hipertensa+espiro y rata Sham (control, operación ficticia). (b). Cuantificación del área de los cardiomiocitos según se describe en Materiales y Métodos. Se grafican los promedios $\pm D E$. * $p<$ 0.05 vs DOCA y DOCA+Espiro, **p< 0.05 vs Sham, \#p<0.05 vs DOCA y Sham (post-ANOVA).

3.- Fibrosis del VI. El contenido de colágeno aumentó un $470 \%$ en las ratas hipertensas DOCA respecto a su grupo control (Figura 2). El tratamiento con espiro disminuyó significativamente el contenido de colágeno de las ratas DOCA en un 71,5\% (Figura 2).

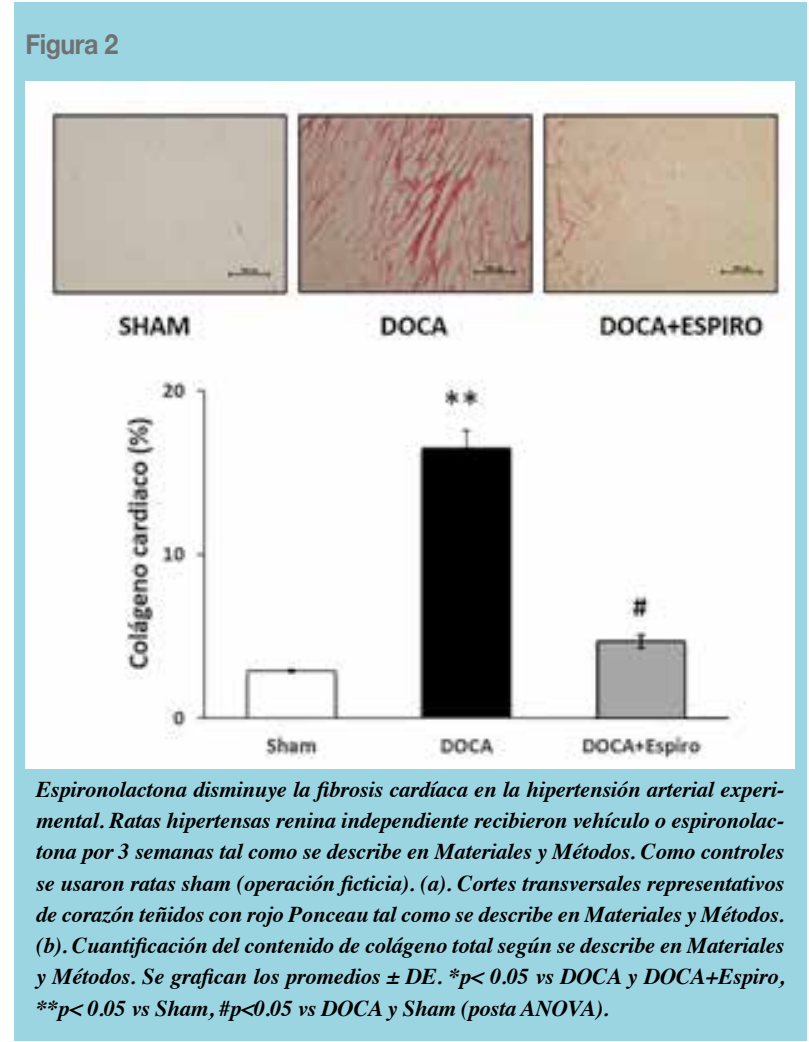

4.- Niveles circulantes de Ang-(1-9). No se encontraron diferencias entre los niveles circulantes de Ang-(1-9) entre las ratas sham y DOCA. Sin embargo, la administración de espiro aumento de manera significativa (430\%) los niveles circulantes de Ang-(1-9) (Figura 3).

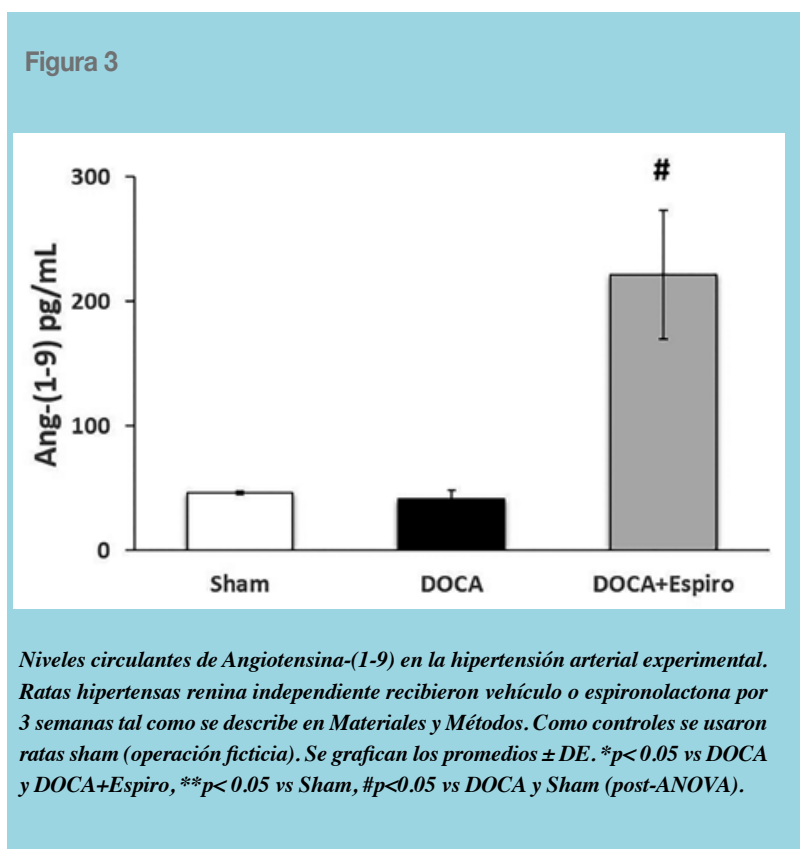


5.- Relación entre área de los cardiomiocitos y niveles circulantes de Ang-(1-9). Estas relaciones se evaluaron correlacionando los niveles circulantes de Ang-(1-9) con el área de los cardiomiocitos de los grupos DOCA y DOCA + espiro (Figura 4). Se observó un nivel de correlación inversa y significativa entre el área de los cardiomiocitos y los niveles circulantes de Ang-(1-9) ( $\mathrm{r}=$ $-0.612, \mathrm{p}<0,05$, Figura 4).

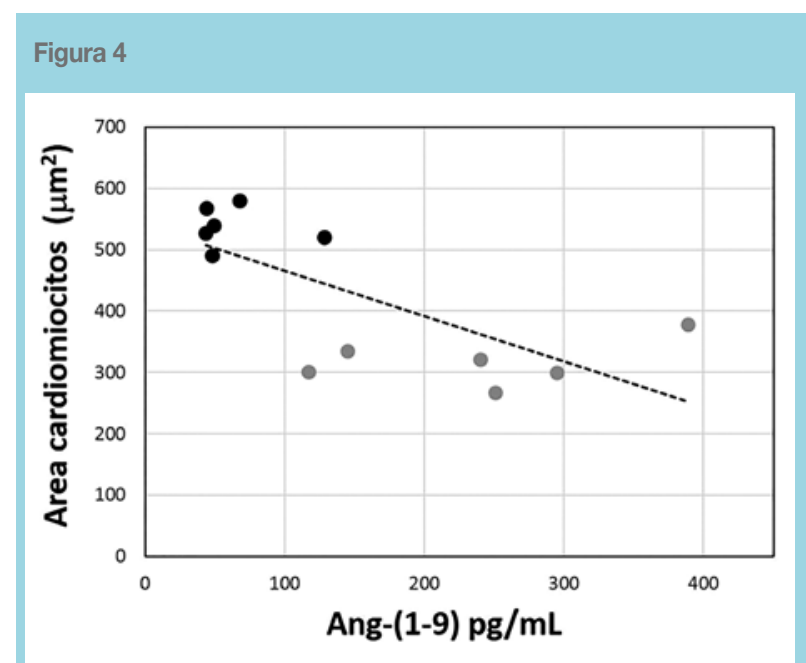

Relación entre los niveles circulantes de Ang-(1-9) y el área de los cardiomiocitos en la hipertensión arterial experimental. Ratas hipertensas renina independiente recibieron vehículo o espironolactona por 3 semanas tal como se describe en Materiales y Métodos. La correlación entre el cambio en el área de los cardiomiocitos y los niveles circulantes de Ang-(1-9) de las ratas DOCA y DOCA+espiro se determinaron simultáneamente $(r=-0.612, p<0,05)$. Para las correlaciones se usó método de Pearson con el programa estadístico SPSS 10.0. Simbología: • ratas DOCA, : ratas DOCA+espiro.

6.- Relación entre fibrosis ventricular izquierda $y$ niveles circulantes de Ang-(1-9) El análisis de la relación entre la fibrosis ventricular izquierda y los niveles plasmáticos de Ang-(1-9) de las ratas DOCA y DOCA+ espiro mostraron correlación inversa y significativa $(\mathrm{r}=$ $-0,607, \mathrm{p}<0,05$, Figura 5).

\section{Discusión:}

El principal resultado de este estudio ha sido el hallazgo de que en un modelo de HTA experimental, la administración del bloqueador del receptor de mineralocorticoide espironolactona, junto con normalizar las cifras de presión arterial, aumentó significativamente (en 4 veces) los niveles circulantes de Ang-(1-9). Esta es la primera observación reportada del efecto de espironolactona sobre los niveles de Ang-(1-9) en hipertensión arterial. El aumento de Ang-(1-9) inducido por espironolactona se

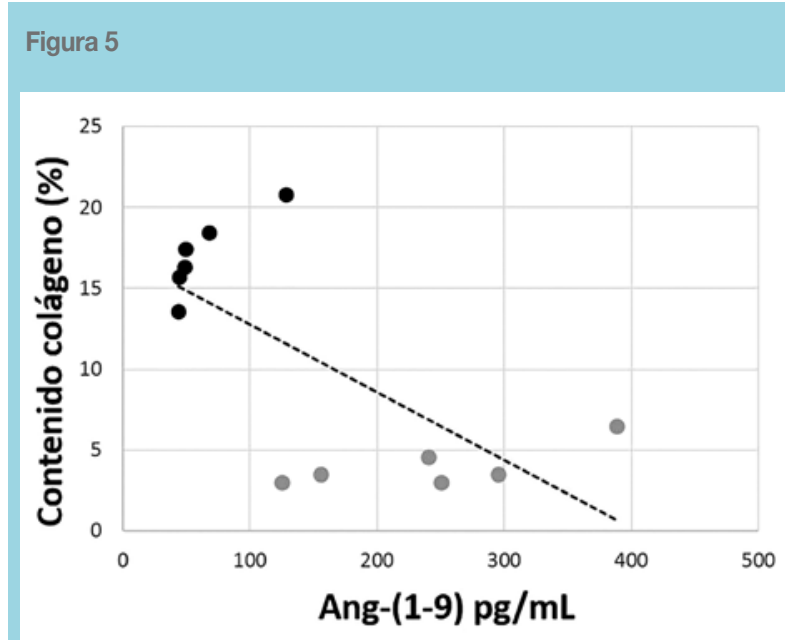

Relación entre los niveles circulantes de Ang-(1-9) y el contenido de colágeno cardiaco en la hipertensión arterial experimental. Ratas hipertensas renina independiente recibieron vehículo o espironolactona por 3 semanas tal como se describe en Materiales y Métodos. La correlación entre el cambio en el contenido de colágeno cardiaco y los niveles circulantes de Ang-(1-9) de las ratas DOCA y DOCA+espiro se determinaron simultáneamente $(r=-0,607, p<0,05)$. Para las correlaciones se usó método de Pearson con el programa estadístico SPSS 10.0. Simbología: • : ratas DOCA, : ratas DOCA+espiro

correlacionó con la regresión del remodelado cardíaco hipertensivo, específicamente hipertrofia $(r=-0.612, \mathrm{p}<$ $0,05)$ y fibrosis cardíaca $(r=0,607, p<0,05)$ en este modelo experimental de HTA severa, efecto conocidos del uso de espironolactona en hipertensión arterial. ${ }^{12}$

Los primeros antecedentes respecto a la participación del eje ECA2-Ang-(1-9) en la regresión del remodelado cardíaco fue aportada por nuestro Laboratorio. ${ }^{9} \mathrm{Al}$ respecto, estudios realizados en el modelo experimental de infarto al miocardio (IAM) a las 8 semanas post-cirugía mostraron un aumento de la actividad de ECA y de los niveles de Ang II y una disminución de la actividad de ECA2 y de los niveles circulantes de Ang-(1-9). ${ }^{9}$ Estos cambios favorecieron la fibrosis miocárdica en el área no infartada medida por la fracción volumétrica de colágeno y el desarrollo de hipertrofia ventricular izquierda (HVI) patológica. ${ }^{9}$ La inhibición de la ECA con enalapril previno la disminución de la actividad de ECA2 y aumentó significativamente los niveles circulantes de Ang-(1-9). ${ }^{9}$ Estos resultados sugirieron una interacción entre ECA y ECA-2 en el remodelado miocárdico post IAM como también que la Ang-(1-9) más que la Ang-(1-7) actuarían como un contra regulador de la Ang II. ${ }^{9}$

Estudios posteriores mostraron también que el tratamiento crónico de ratas infartadas con candesartán aumentó los niveles circulantes de Ang-(1-9). Además solo los niveles circulantes de Ang-(1-9) y no de Ang II y Ang- 
(1-7) correlacionaron de manera inversa con el desarrollo de HVI medida como masa cardíaca relativa y contenido de total de proteínas ventriculares. ${ }^{10}$ Adicionalmente, en este estudio encontramos que mayores niveles circulantes de Ang-(1-9) inducidos por la administración de enalapril o candesartán, se asociaron con menor expresión de marcadores moleculares de hipertrofia tales como niveles proteicos de la cadena pesada de la $b$-miosina (b-MHC) y mRNA del factor natriurético auricular. ${ }^{10} \mathrm{La}$ administración crónica de Ang-(1-9) previno in vivo la hipertrofia cardiomiocitaria post infarto y su acción fue independiente de Ang-(1-7). Ang-(1-9) también previno la hipertrofia la hipertrofia de cardiomiocitos neonatos inducida por norepinefrina y el factor de crecimiento de insulina tipo $1 .^{10}$

Los antecedentes de la participación del eje ECA2/ Ang-(1-9) en la HTA, se encontraron en el modelo experimental de HTA por sobrecarga de volumen (modelo DOCA-sal) y en ratas normotensas controles. ${ }^{13}$ En este modelo experimental, la inhibición de RhoA/Rho-kinasa (mecanismo de señalización intracelular que participa en el remodelamiento $\mathrm{CV}$ y renal patológico como también en la regulación de la PA) por fasudil disminuyó la PA y también aumentó la actividad de ECA2 en plasma y en la pared aórtica. Junto con esto, fasudil disminuyó los niveles plasmáticos de Ang II y aumentó los niveles plasmáticos de Ang-(1-9), sin cambios en los niveles circulantes de Ang-(1-7). ${ }^{13}$ Por lo tanto, este novedoso efecto que la inhibición de RhoA/Rho-kinasa aumenta la expresión de ECA2 y los niveles de Ang-(1-9), podría adicionalmente contribuir al efecto antihipertensivo de los inhibidores de RhoA/Rho-kinasa. En conjunto, estos resultados sugirieron que, en este modelo experimental, la HTA es más dependiente de la actividad de ECA2 y niveles de Ang-(1-9) que de la vía ECA/Ang II.

En forma interesante, en este modelo de HTA experimental que incluye uninefrectomía y administración del mineralocorticoide DOCA, los niveles circulantes de Ang(1-9) fueron similares en las ratas sham y en las ratas hipertensas no tratadas a pesar de marcadas diferencias en los niveles de presión arterial y del remodelado cardíaco en ambos grupos y donde es posible esperar diferencias en los niveles de Ang-(1-9). El hecho de una marcada diferencia en los niveles de Ang-(1-9) entre las ratas sham y las ratas hipertensas tratadas con espironolactona permite plantear, sin poder asegurarlo, la especificidad del efecto de espironolactona para esta respuesta. Hubiera sido muy interesante en este sentido observar el efecto de espironolactona sobre los niveles de Ang-(1-9) en ratas sham. Sin embargo, este trabajo es un subestudio y por lo tanto permite generar hipótesis. Hemos observado preliminarmente en ratas espontáneamente hipertensas (SHR), un modelo más semejante a la HTA esencial en seres humanos, que los niveles circulantes de Ang-(1-9) se encuentran disminuidos respecto a sus controles ratas Wistar Kyoto (datos no publicados) y que aumentan al usar candesartán (datos no publicados).

Aunque no han sido determinados en este estudio, posiblemente los niveles tisulares en miocardio y en pared aórtica de Ang-(1-9) también podrían estar aumentados en el grupo de ratas hipertensas DOCA tratadas con espironolactona.

Recientemente, hemos publicado estudios en 2 modelos experimentales de HTA (infusión de Ang II y modelo Goldblatt, 2 riñones -1 clip). En estos modelos experimentales, la administración crónica de Ang-(1-9) disminuyó significativamente la PAS y el daño cardiovascular hipertensivo al disminuir la hipertrofia del cardiomiocito, fibrosis cardíaca, y estrés oxidativo del ventrículo izquierdo. ${ }^{14}$ Estos efectos de Ang-(1-9) fueron mediados por el receptor tipo 2 de Ang II (RAT2). ${ }^{14,15}$ Resultados de otros investigadores han mostrado que el tratamiento con Ang-(1-9) mejora la cardiomiopatía y atenúa la disfunción miocárdica inducida por estreptozotocina. ${ }^{16}$

El mecanismo de acción propuesto para Ang-(1-9) que pudiera explicar el efecto antihipertensivo y de disminuir el daño cardiovascular es unirse al receptor tipo 2 de Ang II (RAT2) y liberar vasodilatadores endoteliales. ${ }^{14}$ Nuestros estudios funcionales en arterias de resistencia ex vivo mostraron que Ang-(1-9) mantiene la relajación dependiente del endotelio e inducida por acetilcolina en aortas de ratas tratadas con Ang II. ${ }^{14}$ Ang-(1-9) también aumentó los niveles de mRNA aórticos de eNOS, efecto que se asoció a mayores niveles plasmáticos de nitratos. Todos estos efectos de Ang-(1-9) fueron bloqueados por PD123319 (bloqueador del RAT2), indicando que Ang-(1-9) aumenta la biodisponibilidad de óxido nítrico (ON) a través de un mecanismo mediado por RAT2. ${ }^{14}$ Igualmente, la administración de Ang-(1-9) mejoró la vaso relajación y los niveles de $\mathrm{ON}$ en ratas stroke-prone espontáneamente hipertensas. ${ }^{17}$ Flores-Muñoz y cols mostraron que Ang-(1-9) aumentó la expresión de la NADPH oxidasa ${ }^{4,17}$ la cual previamente se asoció con vasodilatación dependiente del endotelio y mediada por ON. ${ }^{17}$ Adicionalmente, Ang-(1-9) también estimuló la secreción del péptido natriurético vía el mecanismo de señalización AT2R/PI3K/Akt/NO/cGMP. ${ }^{18}$ En conjunto con ON, Ang-(1-9) también induce la liberación de ácido 
araquidónico - otro vasodilatador - que pudiera estar implicado en el mecanismo de acción de Ang-(1-9). ${ }^{19}$

Por lo tanto, Ang-(1-9) a través de su RAT2 podría disminuir el remodelamiento cardíaco adverso en la HTA.

Ang-(1-9) es producida por la hidrólisis de la enzima ECA2, una carboxipeptidasa, a partir de Ang I. ${ }^{20}$ Ang(1-9) es degradada por hidrólisis de la enzima ECA, una carboxidipeptidasa, a Ang-(1-7). Estudios de metabolización han mostrado que Ang-(1-9) se hidroliza 18 veces más lento que Ang I y 30\% más lento que Ang-(1-7) permitiendo una mayor biodisponibilidad de Ang-(1-9) al presentar mayor tiempo de vida media que esta última. ${ }^{21}$ Además, se ha encontrado en plasma humano y de rata, que los niveles de Ang-(1-9) son mayores a los de Ang II y que este péptido se acumula en animales tratados con inhibidores de la ECA sugiriendo que los efectos benéficos descritos para Ang-(1-7) podrían favorecerse por la presencia de Ang-(1-9).9,22 Estos antecedentes revelan la potencialidad de Ang-(1-9) para ser utilizado directamente como fármaco o generar un tratamiento farmacológico que active la producción o disminuya la degradación de Ang-(1-9).

Un aspecto muy interesante en relación a los resultados observados en este modelo experimental de HTA inducida por DOCA, con niveles de renina y de aldosterona disminuidos por la expansión de volumen y donde están mejor indicados los diuréticos tiazídicos, espironolactona redujo la PA e indujo regresión del remodelado cardíaco en paralelo con el aumento de los niveles de Ang-(1-9), muy similar a lo que se observó con la presión arterial en el estudio Pathway-2 en pacientes con HTA resistente. ${ }^{7}$ Por tanto, no es posible descartar que tanto el efecto hipotensor como los efectos anti hipertrófico y antifibrótico observados tengan relación con el aumento inducido de Ang-(1-9).

Limitaciones. Al tratarse de un subestudio, como se ha mencionado, existen algunas limitaciones. Habría sido interesante disponer de un grupo sham tratado con espironolactona. Tampoco se puede descartar que el efecto de espironolactona sobre los niveles de Ang-(1-9) sea secundario al efecto hipotensor observado. Sin embargo, en las ratas sham los niveles del péptido fueron significativamente menores, lo que disminuye esa posibilidad. El efecto de un antihipertensivo distinto de espironolactona también podría haber contribuido en este aspecto Por otro lado, el efecto de dosis menores - no antihipertensivas - de espironolactona habría sido útil para idealmente evaluar las dosis del fármaco que logre separar el efecto antihipertensivo.

\section{Conclusiones:}

A pesar de las limitaciones mencionadas de este estudio, los resultados muestran claramente que en este modelo de HTA severa y con remodelado cardiovascular importante, espironolactona reduce la PA en paralelo con el aumento de los niveles circulantes de Ang-(1-9), un efecto previamente no conocido de este poderoso fármaco antihipertensivo y anti remodelado y que posiblemente esté contribuyendo a estos efectos de espironolactona. 


\section{Referencias}

1. ZANCHETTI A. Bottom blood pressure or bottom cardiovascular risk? How far can cardiovascular risk be reduced?. J. Hypertension. 2009; 27:1509-1520.

2. OCARANZA M, JALIL J. Protective Role of the ACE2/Ang(1-9) Axis in Cardiovascular Remodeling. Int J Hypertens. 2012; 2012: 594361.

3. CHEN, Z., TAN, F., ERDOS, E, DEDDISH, P. Hydrolysis of angiotensin peptides by human angiotensin I-converting enzyme and the resensitization of B2 kinin receptors. Hypertension. 2005; 46: 1368-1373.

4. SANTOS R, SIMOES E, SILVA A, MARIC C, SILVA D, MACHADO R, et al. Angiotensin-(17) is an endogenous ligand for the $G$ protein-coupled receptor Mas. Proc Natl Acad Sci U S A 2003; 100: 8258-8263.

5. JACKMAN H, MASSAD M, SEKOSAN M, TAN F, BROVKOVYCH V, MARCIC B, et al. Angiotensin 1-9 and 1-7 release in human heart: role of cathepsin A. Hypertension 2002; 39: 976-981.

6. OCARANZA M, MOYA J, BARRIENTOS V, ALZAMORA R, HEVIA D, MORALES C, et al. Angiotensin-(1-9) reverses experimental hypertension and cardiovascular damage by inhibition of the angiotensin converting enzyme/Ang II axis. J Hypertens. 2014; 32: 771-783.

7. WILLIAMS B, MACDONALD T, MORANT S, WEBB D, SEVER P, MCINNES G, et al. The British Hypertension Society's PATHWAY Studies Group. Spironolactone versus placebo, bisoprolol, and doxazosin to determine the optimal treatment for drug-resistant hypertension (PATHWAY-2): a randomised, double-blind, crossover trial. Lancet 2015; 386: 2059-2068.

8. ARAOS P, MONDACA D, JALIL JE, YAÑEZ C, NOVOA U, MORA I, et al. Diuretics prevent Rho-kinase activation and expression of profibrotic/oxidative genes in the hypertensive aortic wall. Ther Adv Cardiovasc Dis. 2016 Sep 1. pii: 1753944716666208 .

9. OCARANZA M, GODOY I, JALIL J, VARAS M, COLLANTES P, PINTO M, et al. Enalapril attenuates downregulation of Angiotensin-converting enzyme 2 in the late phase of ventricular dysfunction in myocardial infarcted rat. Hypertension 2006; 48: 572-578.

10. OCARANZA MP, LAVANDERO S, JALIL JE, MOYA J, PINTO M, NOVOA U, et al.. Angiotensin-(1-9) regulates cardiac hypertrophy in vivo and in vitro. J Hypertens. 2010; 28 : 1054-64.

11. OCARANZA M, DÍAZ-ARAYA G, CARREÑO J, MUÑOZ D, RIVEROS J, JALIL J, et al. Polymorphism in gene coding for ACE determines different development of myocardial fibrosis in rats. Am J Physiol Heart Circ Physiol. 2004; 286: H498-506.

12. SATO A, HAYASHI M, SARUTA T. Relative long-term effects of spironolactone in conjunction with an angiotensin-converting enzyme inhibitor on left ventricular mass and diastolic function in patients with essential hypertension. Hypertens Res. 2002; 25: 837-842.

13. OCARANZA M, RIVERA P, NOVOA U, PINTO M, GONZÁLEZ L, CHIONG M, et al. Rho kinase inhibition activates the homologous angiotensin-converting enzyme-angiotensin-(1-9) axis in experimental hypertension. J Hypertens. 2011; 29: 706-715.

14. OCARANZA M, MICHEA L, CHIONG M, LAGOS C, LAVANDERO S, JALIL J. Recent insights and therapeutic perspectives of angiotensin-(1-9) in the cardiovascular system. Clin Sci. 2014; 127: 549-557.

15. FLORES-MUÑOZ M, SMITH N, HAGGERTY C, MILLIGAN G, NICKLIN SA. Angiotensin19 antagonises pro-hypertrophic signalling in cardiomyocytes via the angiotensin type 2 receptor. J Physiol. 2011; 589: 939-551. 
16. ZHENG H, PU S, FAN X, LI X, ZHANG Y, YUAN J, et al. Treatment with angiotensin-(1-9) alleviates the cardiomyopathy in streptozotocin-induced diabetic rats. Biochem Pharmacol. 2015; 95: 38-45.

17. FLORES-MUNOZ M, WORK L, DOUGLAS K, DENBY L, DOMINICZAK A, GRAHAM D, et al. Angiotensin-(1-9) attenuates cardiac fibrosis in the stroke-prone spontaneously hypertensive rat via the angiotensin type 2 receptor. Hypertension. 2012; 59: 300-307

18. CHA S, PARK B, GAO S, KIM S. Stimulation of ANP by angiotensin-(1-9) via the angiotensin type 2 receptor. Life Sci. 2013; 93: 934-940.

19. CHENG Z, VAPAATALO H, MERVAALA E. Angiotensin II and vascular inflammation. Med Sci Monit. 2005; 11: RA194-205.

20. DONOGHUE, M., HSIEH, F., BARONAS, E., GODBOUT, K., GOSSELIN, M., STAGLIANO, N, et al. A novel angiotensin-converting enzyme-related carboxypeptidase (ACE2) converts angiotensin I to angiotensin 1-9. Circ. Res. 2000; 87: E1-E9.

21. CHEN Z, TAN F, ERDOS E, DEDDISH P. Hydrolysis of angiotensin peptides by human angiotensin I-converting enzyme and the resensitization of B2 kinin receptors. Hypertension. 2005; 46: 1368-1373.

22. JOHNSON H, KOURTIS S, WATERS J, DRUMMER O. Radioimmunoassay for immunoreactive [des-Leu10]- angiotensin. Peptides. 1989; 10: 489-492. 